\title{
A Compact Two-Frequency Notch Filter for Millimeter Wave Plasma Diagnostics
}

\author{
D. Wagner ${ }^{1}$ (D) $\cdot$ W. Kasparek ${ }^{2} \cdot$ F. Leuterer ${ }^{1} \cdot$ F. Monaco ${ }^{1} \cdot$ T. Ruess $^{3} \cdot$ J. Stober $^{1}$. $^{-}$ \\ M. Thumm ${ }^{3}$
}

Received: 18 March 2020 / Accepted: 6 May 2020 / Published online: 27 May 2020

(C) The Author(s) 2020

\begin{abstract}
Sensitive millimeter wave diagnostics in magnetic confinement plasma fusion experiments need protection from gyrotron stray radiation in the plasma vessel. Modern electron cyclotron resonance heating $(\mathrm{ECRH})$ systems take advantage of multifrequency gyrotrons. This means that the frequency band of some millimeter wave diagnostics contains more than one narrow-band gyrotron-frequency line, which needs to be effectively suppressed. A compact standard waveguide notch filter based on coupled waveguide resonators with rectangular cross-section is presented which can provide very high suppression of several gyrotron frequencies and has low insertion loss of the passband.
\end{abstract}

Keywords Electron cyclotron resonance heating · Magnetically confined fusion plasmas · Millimeter wave diagnostics $\cdot$ Notch filter

\section{Introduction}

Modern multi-frequency megawatt-class gyrotrons can operate at several frequencies in the millimeter wave range [1-4]. These frequencies correspond to the various transmission maxima of their single-disk chemical vapor deposition (CVD) diamond window. In recent years, high-power gyrotrons became very attractive as sources for electron cyclotron resonance heating (ECRH) systems in thermonuclear fusion plasma experiments, since they allow for more flexibility with respect to the applied magnetic field in the magnetic confinement fusion devices [5]. Sensitive

\section{Wagner}

dietmar.wagner@ipp.mpg.de

1 Max-Planck-Institut für Plasmaphysik, Boltzmannstr.2, D-85748 Garching, Germany

2 Institut für Grenzflächenverfahrenstechnik und Plasmatechnologie, Universität Stuttgart, Pfaffenwaldring 31, D-70569 Stuttgart, Germany

3 Karlsruher Institut für Technologie, Institut für Hochleistungsimpuls- und Mikrowellentechnik, Kaiserstr. 12, D-76131 Karlsruhe, Germany 
Fig. 1 Geometry of the stepped waveguide width (top), frequency characteristic of reflected (middle) and transmitted modes (bottom)
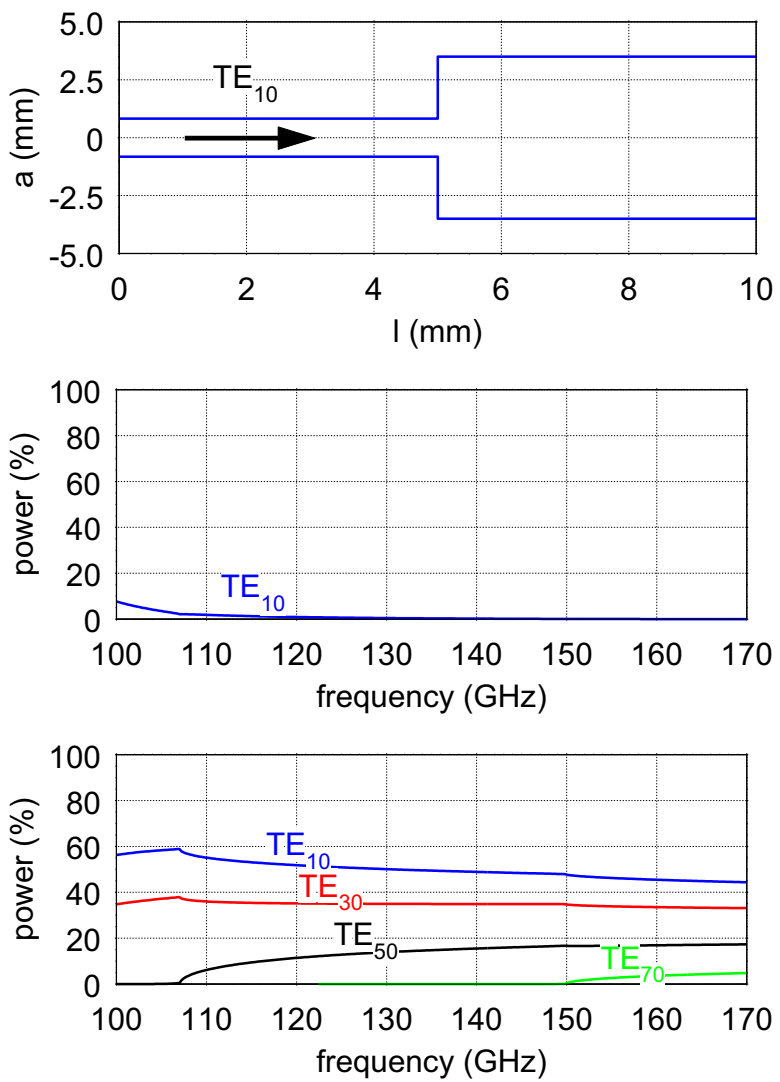

millimeter wave diagnostic systems need protection against ECRH stray radiation [6]. When applying multi-frequency gyrotrons, more than one frequency must be suppressed. A specific problem concerning these filters is the frequency chirp of high-power gyrotrons due to cavity heating and expansion, specifically at the beginning of the pulse, which can range from tens to

Fig. 2 Calculated transmitted power as a function of width $a_{\mathrm{r}}$ and length $l_{\mathrm{r}}$ of the waveguide cavity. The red lines mark the domains where the transmitted power is below $-23 \mathrm{~dB}$. The arrow marks the point where the minima for both frequencies meet and therefore gives the correct dimensions of the desired cavity $\left(a_{\mathrm{r}}=6.72 \mathrm{~mm}, b_{\mathrm{r}}=0.826 \mathrm{~mm}, l_{\mathrm{r}}=\right.$ $1.68 \mathrm{~mm}$ )

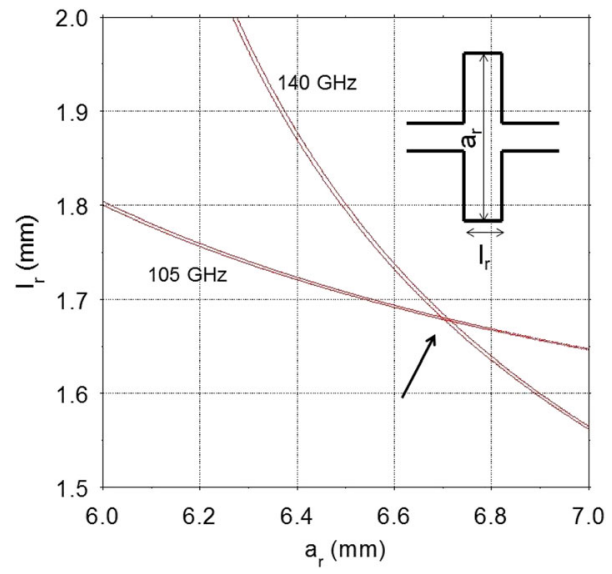


Fig. 3 Calculated transmission of the $\mathrm{TE}_{10}$-mode of a single resonator with the dimensions $a_{\mathrm{r}}=$ $6.72 \mathrm{~mm}, b_{\mathrm{r}}=0.826 \mathrm{~mm}, l_{\mathrm{r}}=1.68$ $\mathrm{mm}$

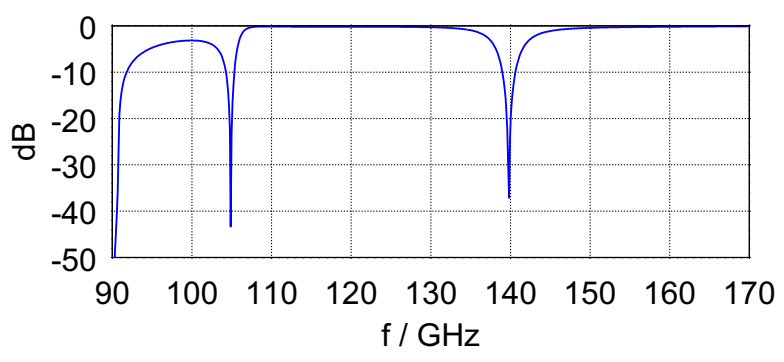

hundreds of MHz [7]. For ECRH systems applying many different gyrotrons, the notches need to be at least as wide as the specified accuracy provided by the gyrotron manufacturer, which is typically \pm $250 \mathrm{MHz}$. These requirements are hard to fulfill with the available filter technology. Tunable coupled-cavity filters are difficult to be tuned for more than one specific stopband. Multi-frequency filters like optical Fabry-Perot resonance filters can provide various but only very narrow notches [6]. In principle, it is possible to switch from one single frequency notch filter to another one, depending on the applied gyrotron frequency, or to connect two single frequency notch filters in series, with the latter leading to an increase in the overall insertion loss. However, for application of two gyrotron frequencies, it is preferable to have a single filter providing two stopbands. Two-

Fig. 4 Resonator geometry (top) and normalized modal amplitude distributions at the resonance frequencies $105 \mathrm{GHz}$ (middle) and $140 \mathrm{GHz}$ (bottom)
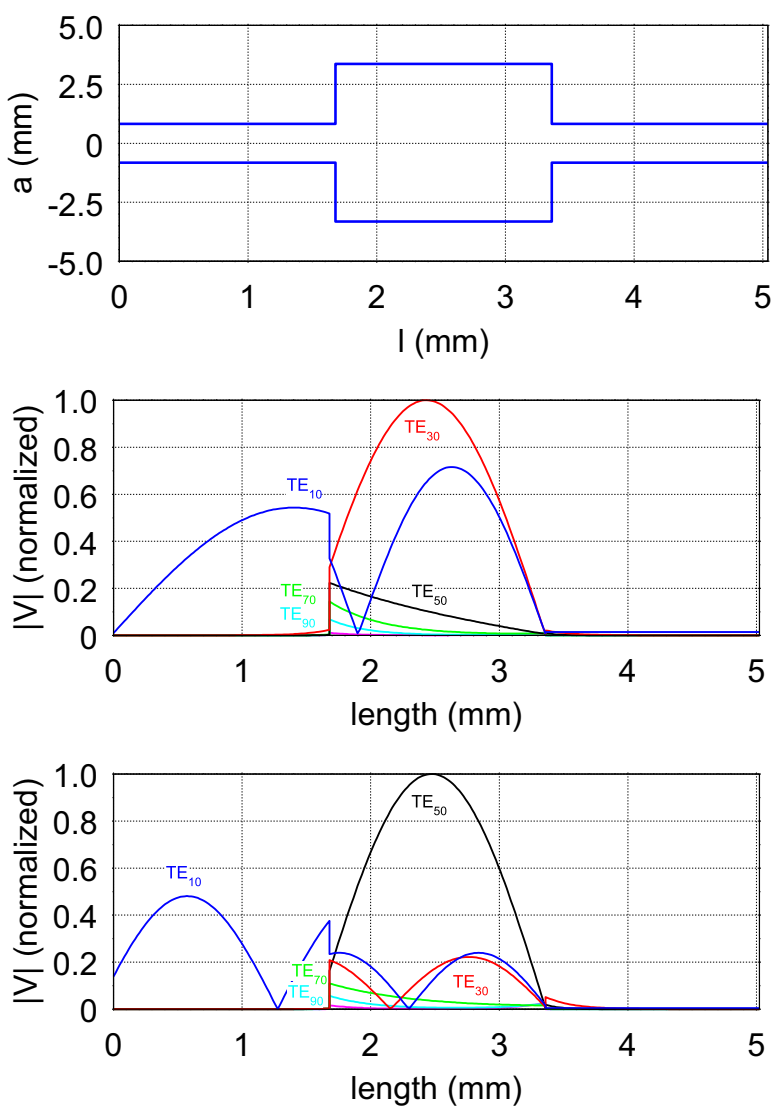
Fig. 5 Calculated electric field distribution at the resonance frequencies $105 \mathrm{GHz}$ (top) and $140 \mathrm{GHz}$ (bottom)
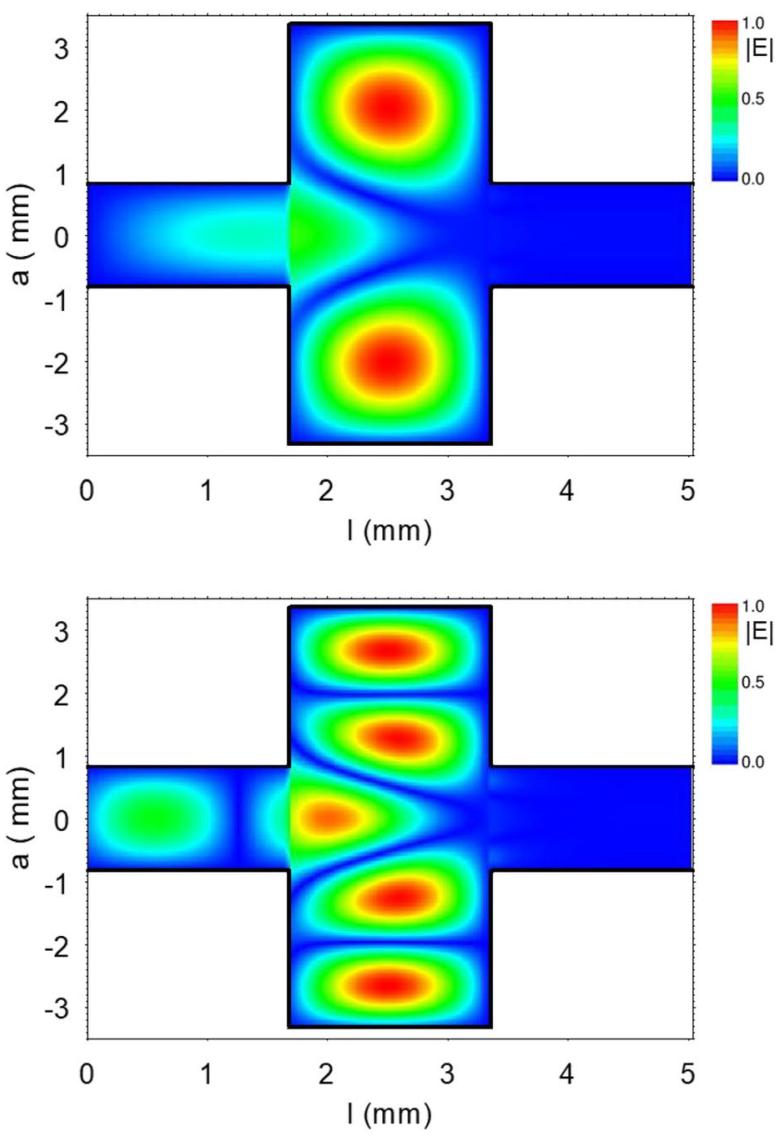

frequency notch filters for the electron cyclotron emission (ECE) diagnostic systems both at the ASDEX Upgrade tokamak experiment at IPP Garching and the W7-X stellarator at IPP Greifswald were successfully realized applying advanced waveguide Bragg reflectors [8, 9]. In the present paper, a much simpler and compact approach based on standard waveguide resonators with rectangular cross-section [10-13] is presented. The filter described in this paper is designed for application in ECE systems which are operated over a wide frequency range. The width of the stopbands is not critical for ECE as long as they are wide enough to suppress all gyrotron frequencies of the ECRH system. There are other diagnostics, e.g., collective Thomson scattering systems,

Fig. 6 Two-frequency notch filter geometry with 5 cavities

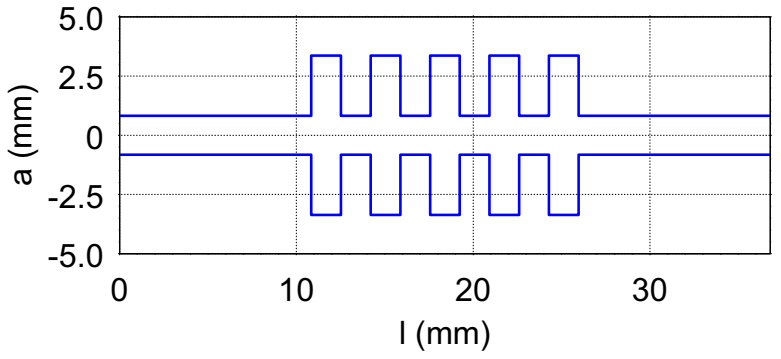


Fig. 7 Comparison of the calculated transmission of the $\mathrm{TE}_{10}$-mode of a single $(n=1)$ up to a chain of $n=5$ resonators with the dimensions $a_{\mathrm{r}}=6.72 \mathrm{~mm}, b_{\mathrm{r}}=$ $0.826 \mathrm{~mm}$, and $l_{\mathrm{r}}=1.68 \mathrm{~mm}$. The distance between the cavities is $1.68 \mathrm{~mm}$

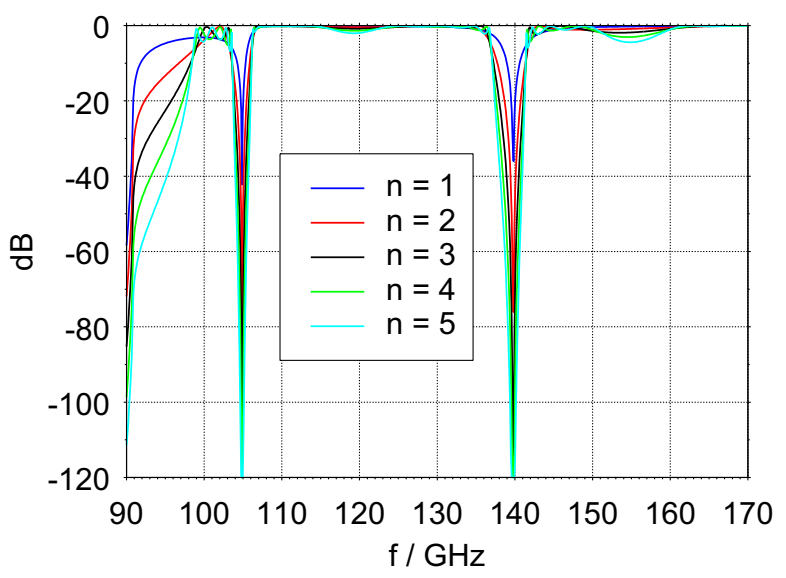

which would require a design with much narrower notches in order to allow for measurements very close to the gyrotron frequencies, but with no interest in wide passbands.

\subsection{Filter Design}

The goal of the present notch filter design is to reject the two frequencies of the ASDEX Upgrade and W7-X ECRH systems, which are 105 and $140 \mathrm{GHz}$. The filter should have no additional stopbands over the full waveguide band (D-band). This can be realized with inwaveguide resonators as described in [9], i.e., standard rectangular waveguides with symmetric steps in the width. In this case, there is no coupling to $\mathrm{TM}$ modes and an incident $\mathrm{TE}_{1,0}$ fundamental waveguide mode only couples to $\mathrm{TE}_{2} \cdot \mathrm{m}+1,0$ modes providing a very limited mode spectrum. To calculate the step-type coupling, a mode matching formalism [9] is applied, taking into account both propagating and evanescent modes. As an example, Fig. 1 shows the coupling of a symmetric step from fundamental waveguide with width $a=1.651 \mathrm{~mm}$ (WR-06)
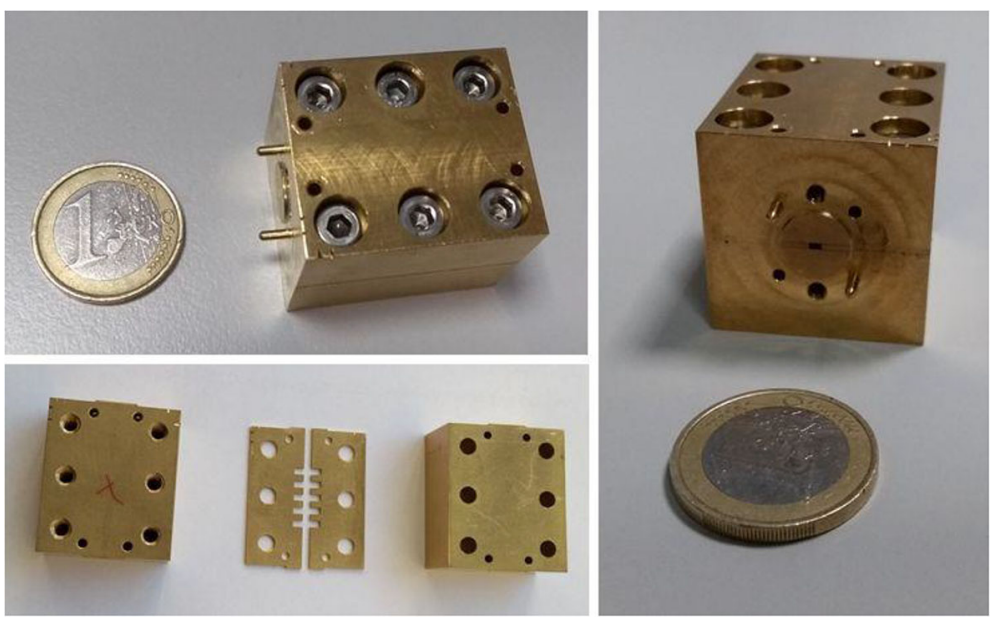

Fig. 8 Photos of the 5-cavity filter 
Fig. 9 Measured and calculated frequency characteristics of the two-frequency notch filter before and after specific surface treatments

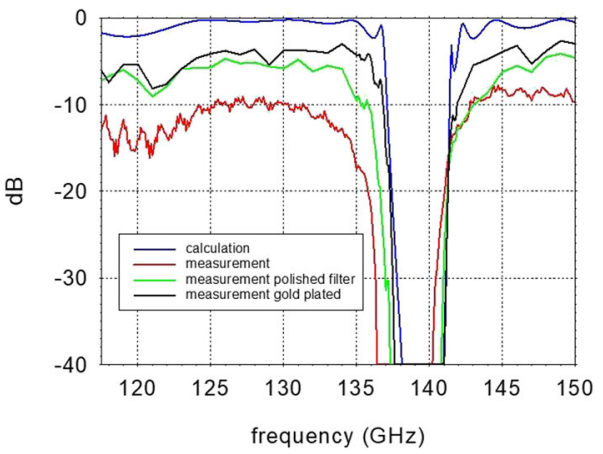

to $a_{\mathrm{r}}=7.0 \mathrm{~mm}$ and with a constant height $b=0.826 \mathrm{~mm}$. Due to symmetry, the incident $\mathrm{TE}_{10}$ mode only couples to $\mathrm{TE}_{\mathrm{m} 0}$ modes with odd $\mathrm{m}=3,5,7 \ldots$.

A resonant cavity at both 105 and $140 \mathrm{GHz}$ can be created by a stepped waveguide where the length $l_{\mathrm{r}}$ of the step corresponds to approximately half of the wavelength for the $\mathrm{TE}_{30}$ mode at $105 \mathrm{GHz}$ and the $\mathrm{TE}_{50}$ mode at $140 \mathrm{GHz}$. The optimum dimensions of the waveguide cavity can be determined by calculating the power transmission at both frequencies as a function of both the width $a_{\mathrm{r}}$ and the length $l_{\mathrm{r}}$ of a single cavity. Figure 2 shows the transmission minima at both frequencies. The point where both domains meet gives the correct dimension of the desired cavity.

Figure 3 shows the calculated frequency characteristic of the resonator with only two notches over the full D-Band. The modal amplitude distributions along the waveguide circuit at the 105 and $140 \mathrm{GHz}$ resonances are plotted in Fig. 4.

As can be seen from Fig. 4, there exist resonant mode mixtures with the dominant modes being $\mathrm{TE}_{30}$ at $105 \mathrm{GHz}$ and $\mathrm{TE}_{50}$ at $140 \mathrm{GHz}$, but also evanescent $\mathrm{TE}_{\mathrm{m} 0}$ cutoff modes have to be taken into account in the generalized scattering matrix calculations. The resonant electric field distributions over the horizontal waveguide cross-section at the two resonances are plotted in Fig. 5.

\subsection{Multi-Cavity Filter}

The signal suppression of the two stopbands can be further increased by combining several cavities. Figure 6 shows the geometry of a filter with 5 resonators, where each cavity has the

Fig. 10 Measured and calculated frequency characteristics of the two-frequency notch filter

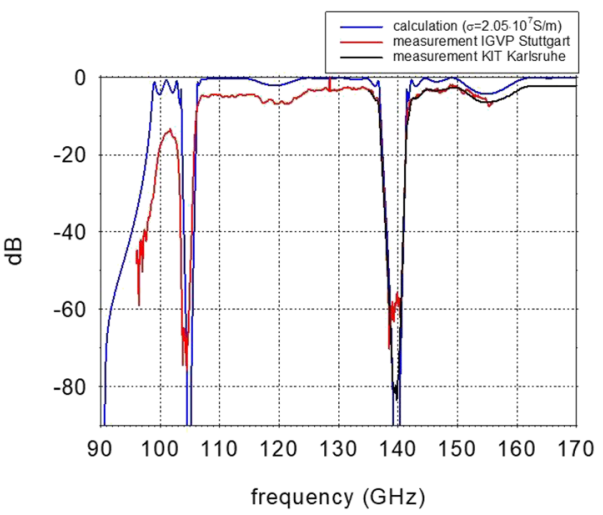


Fig. 11 Measured and calculated frequency characteristics of the two-frequency notch filter in the frequency band from 100 to $110 \mathrm{GHz}$. The dashed lines indicate the operating frequency range of the gyrotrons

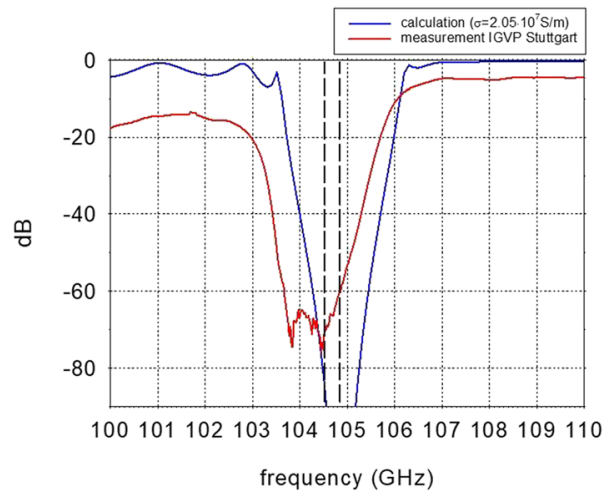

same dimensions as the single cavity investigated in Section 1.1. Figure 7 presents a comparison of the frequency characteristics of filters with a different number of cavities. Both the width of the stopbands and the steepness of the slopes increase with an increasing number of cavities. The transmission coefficient in the frequency range below $110 \mathrm{GHz}$, which is below the D-band and close to cutoff for the propagating TE10 mode, shows a strong dependence on the varying reflection. This is probably due to the varying standing wave ratio in the waveguide.

With 5 cavities, the theoretical notch depth at both resonant frequencies (105 and $140 \mathrm{GHz})$ is below $-120 \mathrm{~dB}$.

\subsection{Measurements}

A 5-cavity filter was fabricated in split-block technology and tested. The filter geometry has been cut out of a sheet metal plate with thickness $0.826 \mathrm{~mm}$, corresponding to the height of a standard WR-06 waveguide. The plate has been sandwiched between two metallic blocks to form the filter geometry. Figure 8 shows photos of the filter and its separate parts. The material is brass. First measurements with the assembled notch filter were performed in the frequency band 117.5-150 GHz using a GYCOM GGBWO-78/178 BWO as source and an ELMIKA D-band detector diode as receiver (Fig. 9). The first measurement revealed a higher insertion loss of about $10 \mathrm{~dB}$ compared with theory as well as a broadened and downshifted upper notch around $140 \mathrm{GHz}$ (red curve). After polishing

Fig. 12 Measured and calculated frequency characteristics of the two-frequency notch filter in the frequency band 135 to $145 \mathrm{GHz}$. The dashed lines indicate the operating frequency range of the gyrotrons

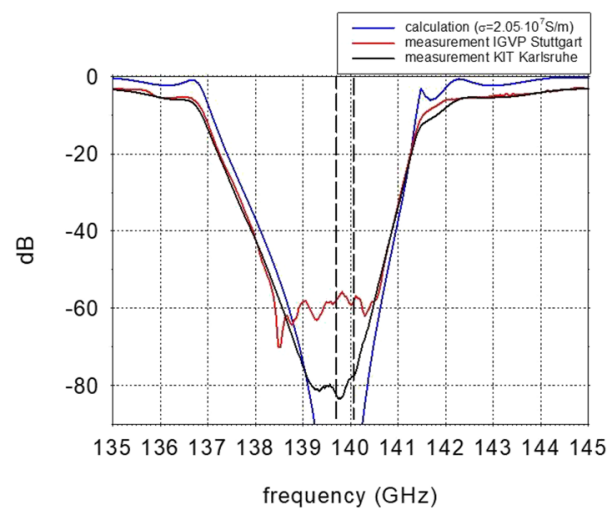


and reassembling of the filter parts, the insertion loss was reduced by $5 \mathrm{~dB}$ and the width of the stopband narrowed (green curve). To further reduce the surface losses and improve the contact between the center plate and both upper and lower block, all the parts were gold plated and assembled again. The measurement with the gold plated filter is given by the black curve, which is again closer to the theoretical values (blue curve). Since the dynamic range of these measurements was only about $-40 \mathrm{~dB}$, we performed additional measurements at IGVP Stuttgart using an ABmm 8-350 Vector Network Analyzer (VNA) in the frequency range from 96 to $156 \mathrm{GHz}$ and at KIT Karlsruhe using a PNA N5222B VNA from 135 to $170 \mathrm{GHz}$. These measurements are shown in Fig. 10 together with the theoretical frequency characteristic. Both measurements are in excellent agreement and their frequency dependence compares well with the calculation. The dynamic range of the ABmm VNA is limited to about $-60 \mathrm{~dB}$ around $140 \mathrm{GHz}$ where its background noise dominates, while the dynamic range of the KIT-measurement was $-80 \mathrm{~dB}$. Half of the conductivity of ideal gold was assumed in the calculation to cope for surface roughness. However, the measured insertion loss is still somewhat higher than calculated ( $3-4 \mathrm{~dB}$ in the range $110-140 \mathrm{GHz}$ and about $2 \mathrm{~dB}$ between 140 and $170 \mathrm{GHz}$ ). This is most probably due to non-perfect contact between the two blocks and the center sheet over the common surface in this split block construction, also leading to a downshift of the resonance frequencies (see also Fig. 9). Figures 11 and 12 give more details in the two rejection bands around 105 and $140 \mathrm{GHz}$. The frequency range of all 8 ASDEX Upgrade gyrotrons, including their frequency chirps and drifts during the pulse, is indicated by the dashed lines. All gyrotron frequencies are within the stopbands.

Summary A compact rectangular waveguide two-frequency notch filter has been designed, fabricated, and tested. The filter provides suppression of two narrow frequency intervals in the D-band. This could be realized by introducing symmetric steps in the width of a standard WR06 waveguide. The measured notch depth is about $-60 \mathrm{~dB}$ at $105 \mathrm{GHz}$ and $-80 \mathrm{~dB}$ at $140 \mathrm{GHz}$. No additional tuning was required.

Acknowledgments The authors would like thank H. Schütz and G. Grünwald from IPP Garching for support with the construction, assembling, and measurement of the notch filter. We are also indebted to B. Graf and M. Father from IPP for helpful discussions and the precise manufacturing.

Funding Information Open access funding provided by Projekt DEAL.

Open Access This article is licensed under a Creative Commons Attribution 4.0 International License, which permits use, sharing, adaptation, distribution and reproduction in any medium or format, as long as you give appropriate credit to the original author(s) and the source, provide a link to the Creative Commons licence, and indicate if changes were made. The images or other third party material in this article are included in the article's Creative Commons licence, unless indicated otherwise in a credit line to the material. If material is not included in the article's Creative Commons licence and your intended use is not permitted by statutory regulation or exceeds the permitted use, you will need to obtain permission directly from the copyright holder. To view a copy of this licence, visit http://creativecommons.org/licenses/by/4.0/.

\section{References}

1. Nichiporenko, V.O., et al., State of the Art of $1 \mathrm{MW} / 105-140 \mathrm{GHz} / 10 \mathrm{sec}$ Gyrotron Project in GYCOM. Conf. Digest 31st Int. Conf. on Infrared and Millimeter Waves and 14th Int. Conf. on Terahertz Electronics, Shanghai, China, 338 (2006). 
2. Thumm, M., et al., EU Megawatt-Class 140-GHz CW Gyrotron, IEEE Trans. on Plasma Science, Vol. 35, 143-153 (2007).

3. Ikeda, R., et al., Multi-Frequency, MW-Power Triode Gyrotron having a Uniform Directional Beam, J Infrared Milli Terahz Waves, Vol. 38, 531-537 (2017).

4. Marchesin, R., et al., Manufacturing and Test of the 1 MW Long-Pulse 84/126 Dual-Frequency Gyrotron for TCV. 20th IEEE Int. Vacuum Electronics Conf. (IVEC 2019), Busan, South Korea, 13.4 (2019).

5. D. Wagner et al., Status, Operation and Extension of the ECRH System at ASDEX Upgrade, International Journal of Infrared, Millimeter and Terahertz Waves, Vol. 37, 45-54 (2016).

6. P. Woskov, Notch Filter Options for ITER Stray Gyrotron Radiation, Proceedings 49th Annual Meeting of the Division of Plasma Physics, Orlando, Florida, 52, No.11, NP8.00111 (2007).

7. D. Wagner et al., Status of the new multi-frequency ECRH System for ASDEX Upgrade, Nuclear Fusion, Vol. 48, 054006 (6pp) (2008).

8. D. Wagner et al., A Multifrequency Notch Filter for Millimeter Wave Plasma Diagnostics based on Photonic Bandgaps in Corrugated Circular Waveguides, EPJ Web of Conferences, Vol. 87, 04012 (2015).

9. D. Wagner, J. Pretterebner, M. Thumm, Transverse Resonances in Oversized Waveguides, Proc. 18th Intl. Conf. on Infrared and Millimeter Waves, Colchester, U.K., W6.6 (1993).

10. G.G. Denisov, S.V. Kuzikov, and D.A. Lukovnikov, Simple Millimeter Wave Notch Filters Based on Rectangular Waveguide, International Journal of Infrared and Millimeter Waves, Vol. 16, No.7, 1231-1238 (1995).

11. G.G. Denisov, et al., Design and Test of New Millimeter Wave Notch Filter for Plasma Diagnostics, Proc. 33rd Intl. Conf. on Infrared, Millimeter and Terahertz Waves, Pasadena, USA (2008), https://doi. org/10.1109/ICIMW.2008.4665485.

12. Y.Y. Danilov et al., Millimeter-Wave Tunable Notch Filter Based on Waveguide Extension for Plasma Diagnostics, IEEE Transactions on Plasma Science, Vol. 42, No. 6, 1685-1689 (2014).

13. D. Wagner et al., Bragg Reflection Band Stop Filter for ECE on Wega, International Journal of Infrared, Millimeter and Terahertz Waves, Vol. 32, 1424-1433 (2011).

Publisher's Note Springer Nature remains neutral with regard to jurisdictional claims in published maps and institutional affiliations. 